\title{
Small reduction of capsaicin-induced neurogenic inflammation in human forearm skin by the glucocorticoid prednicarbate
}

\author{
R. Tafler ${ }^{1}$, M. K. Herbert ${ }^{1, *}$, R. F. Schmidt ${ }^{2}$ and K. H. Weis ${ }^{1}$ \\ ${ }^{1}$ Institut für Anaesthesiologie der Universität Würzburg, Josef-Schneider-Straße 2, and ${ }^{2}$ Physiologisches Institut der Universität \\ Würzburg, Röntgenring 9, D-8700 Würzburg, Germany
}

\begin{abstract}
Capsaicin applied to human skin provokes a response known as neurogenic inflammation. Neuropeptides (substance P, CGRP), released from afferent C-fiber terminals and histamine, secondarily released from mast cells, are supposed to participate in this reaction. We investigated the contribution of arachidonic acid and metabolic products to neurogenic inflammation, using a potent topically applied glucocorticoid and the corresponding vehicle. Arachidonic acid is liberated from membrane phospholipids by phospholipase $\mathrm{A}_{2}$, an enzyme that can be blocked by glucocorticoids. In 12 healthy volunteers, neurogenic inflammation was induced by capsaicin $1 \%$ on both upper forearms after $16 \mathrm{~h}$ of topical pretreatment with either prednicarbate or vehicle. Neurogenic inflammation was assessed by laser Doppler flowmetry and by planimetry of flare sizes. Prednicarbate significantly reduced the laser Doppler flow values inside the flare responses, as well as the flare sizes themselves. These results show that to some extent glucocorticoids reduce capsaicin-induced neurogenic inflammation.
\end{abstract}

\section{Introduction}

Capsaicin application to the skin causes vasodilatation extending beyond the area of application. This response is called neurogenic inflammation. It is supposed that the visible redness (flare) is due to axon reflex vasodilatation via primary afferent neurones (C-fibers), having an efferent function, i.e. liberating neuropeptides (e.g. substance P, CGRP) from their terminals [1]. Besides these neuropeptides, other endogenous vasoactive agents have been suspected to participate in neurogenic inflammation. The present study was undertaken in order to assess the eventual contribution of arachidonic acid to capsaicin-induced neurogenic inflammation. Arachidonic acid is liberated from membrane phos-

* Author for correspondence. pholipids by phospholipase $A_{2}$. This process is inhibited by glucocorticoids in a complex manner [2]. This report is addressed to the following questions:

1. What is the effect of topically applied glucocorticoid on capsaicin-induced neurogenic inflammation in human forearm skin?

2. Does the inhibition of arachidonic acid liberation from membrane phospholipids influence capsaicin-induced neurogenic inflammation?

\section{Materials and methods}

Pretreatment with prednicarbate cream and vehicle cream

After obtaining informed consent, 12 healthy volunteers (aged 22-31 yr) participated in this 
study, which was approved by the local ethics committee. Using a randomized schedule for the choice between left and right side, $3 \mathrm{~g}$ prednicarbate containing cream (Dermatop ${ }^{\circledR}$, Casella Riedel) and $3 \mathrm{~g}$ vehicle cream (Dermatop ${ }^{\circledR}$ Basiscreme, Casella Riedel), respectively, were applied to square areas $\left(6 \times 6 \mathrm{~cm}^{2}\right)$ on both the upper forearms. The cream layers were covered with occlusive dressings overnight for $16 \mathrm{~h}$. Two hours after removal of the cream bandages, the skin was pretreated with DMSO $10 \%$ (Merck) for $1 \mathrm{~h}$ to improve capsaicin absorption.

\section{Induction and assessment of capsaicin-induced} neurogenic inflammation

Neurogenic inflammation was induced by $100 \mu \mathrm{l}$ capsaicin $1 \%$ (Serva) soaked up in a plaster (Hansaplast), $13 \times 18 \mathrm{~mm}^{2}$, applied to the skin for $25 \mathrm{~min}$. The extent of the skin reaction to capsaicin was assessed by measuring the superficial cutaneous blood flow (SCBF) with laser Doppler flowmetry (LDF) (Periflux PF3, PERIMED, Sweden), and by planimetry of flare sizes, i.e. the local visible red- ness. A special holder for the LDF probe was used defining the positions of 12 measuring points on the skin: 4 measuring points inside the application area reflecting the direct action of the substance capsaicin plus an eventual neurogenic inflammation (inside SCBF); 8 peripheral measuring points demonstrating the effects induced by neurogenic inflammation only (outside SCBF). Inside SCBF and outside SCBF were calculated as the means of the respective 4 central and 8 peripheral LDF values. In both series SCBF at rest were subtracted from the test result. All results are expressed as mean \pm SD. The "Wilcoxon signed rank test" was used for statistical analyses (significance level: $p<0.05$ ).

\section{Results}

SCBF following prednicarbate pretreatment and vehicle pretreatment were not significantly different before capsaicin application $(8.6 \pm 1.2$ perfusion units (p.u.); $9.9 \pm 2.3$ p.u.). After capsaicin application, the prednicarbate pretreated side showed a significant reduction of inside SCBF of $18 \%$ (72.0 \pm 13.3 p.u.; $62.7 \pm 16.8$ p.u., Fig. 1). Outside SCBF

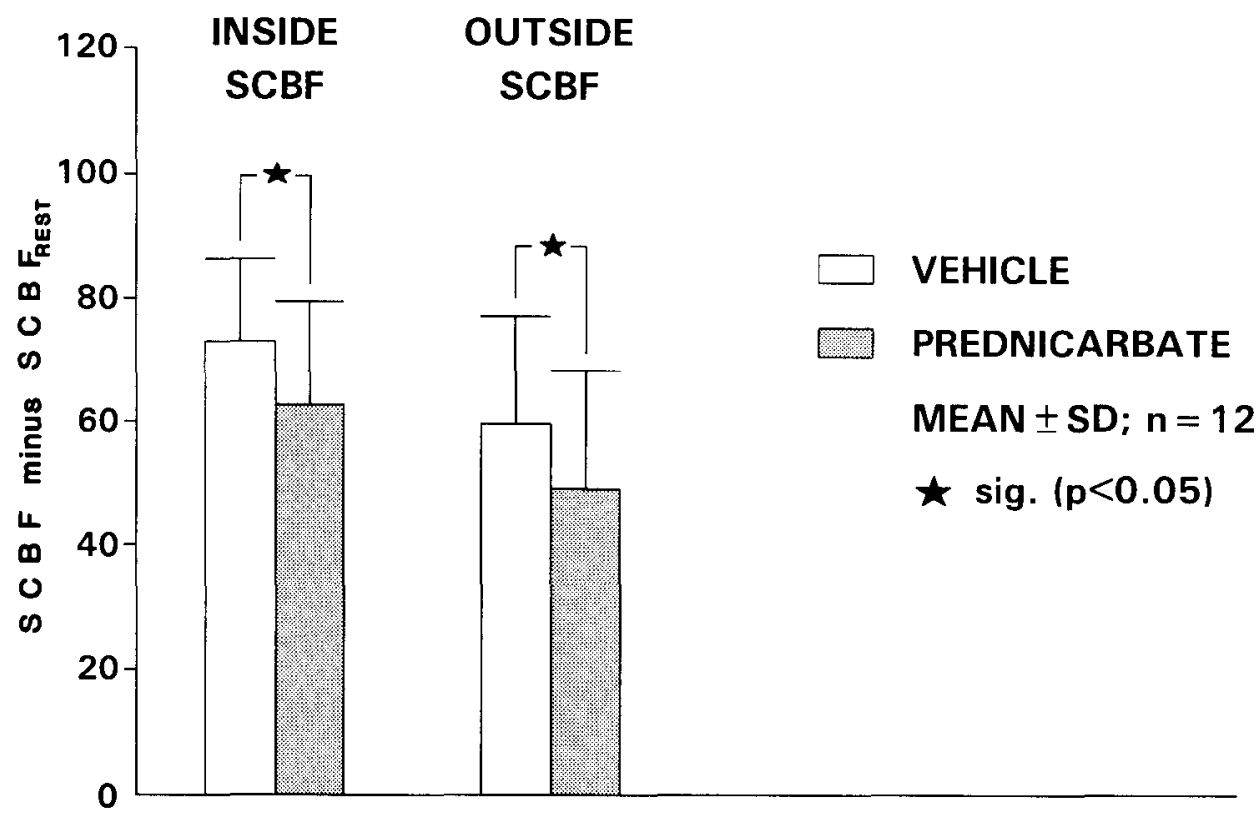

Figure 1

The effect of prednicarbate pretreatment versus vehicle pretreatment on superficial cutaneous blood flow (SCBF) of capsaicin-induced neurogenic inflammation. Inside SCBF reflects the direct effects of the capsaicin plus any additional neurogenic inflammation; outside $\mathrm{SCBF}$ is an exclusive measure of neurogenic inflammation. Ordinate scale is in relative units of perfusion. 


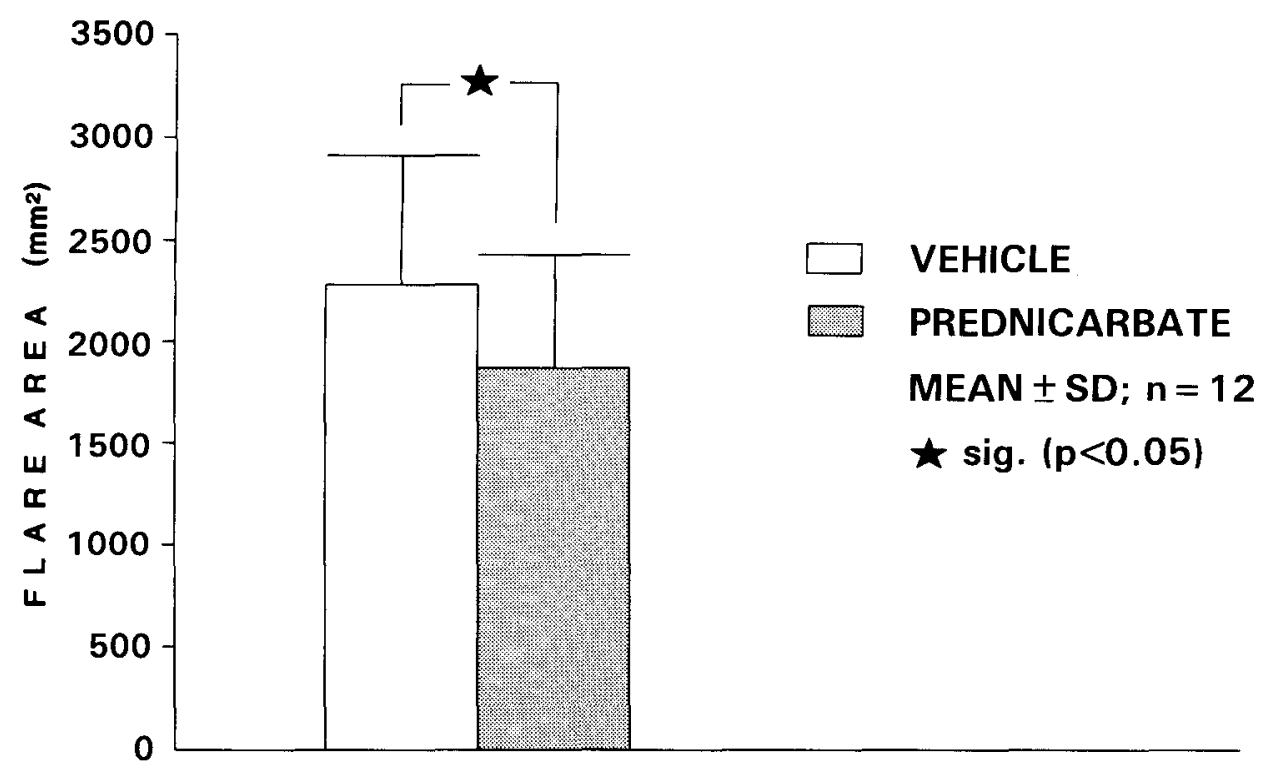

Figure 2

The effect of prednicarbate pretreatment versus vehicle pretreatment on the size of capsaicin-induced flare reaction. Prednicarbate reduced the flare sizes significantly by $18 \%$.

was also significantly reduced by $18 \%$ following prednicarbate pretreatment $(59.6 \pm 17.5$ p.u.; 49.1 \pm 19.0 p.u., Fig. 1). Further, prednicarbate reduced the size of flares significantly by $18 \%$ compared to vehicle $\left(2280.1 \pm 632.4 \mathrm{~mm}^{2} ; 1873.7 \pm 555.0 \mathrm{~mm}^{2}\right.$, Fig. 2).

\section{Discussion}

The small but statistically insignificant difference between SCBF at rest on the corticoid pretreated side versus the vehicle side is in accordance with Bisgaard et al. [3]. It has been shown that glucocorticoids are capable of reducing the endogenous release of arachidonic acid by inhibition of phospholipase $\mathrm{A}_{2}$ [4]. Glucocorticoids are also suggested to inhibit the synthesis of prostaglandins by inhibition of prostaglandin synthase enzyme expression and by coupling lipocortin to membrane phospholipids, in this way reducing the liberation of arachidonic acid [2]. In this study, prednicarbate diminished neurogenic inflammation (flare size as well as $\mathrm{SCBF}$, both significantly by $18 \%$ ). In investigations described elsewhere in these proceedings, we have shown that treatment with acetylsalicylic acid or indomethacin, i.e. a presumed blockade of prostaglandin synthetase, had no effect on capsaicin-induced neurogenic inflammation. The effectiveness of a glucocorticoid and the ineffectiveness of ASA/indomethacin indicates that arachidonic acid or related metabolic products other than those derived from cyclooxygenase pathway may be involved in the development of neurogenic inflammation. Another mode of action of the glucocorticoids could also explain our results, namely their inhibition of the expression of $\mathrm{Ca}^{2+}$-independent $\mathrm{NO}$ synthase, assuming that the potent endogenous vasodilator NO does participate in neurogenic inflammation. This line of reasoning derives from the finding that, in contrast to constitutive $\mathrm{Ca}^{2+}$. dependent NO synthase, the expression of $\mathrm{Ca}^{2+}$-independent $\mathrm{NO}$ synthase is inhibited by glucocorticoids [5].

\section{References}

[1] J. C. Foreman, The skin as an organ for the study of the pharmacology of neuropeptides. Skin Pharmacol. 1, 77-83 (1988).

[2] J. M. Bailey, New mechanisms for effects of anti-inflammatory glucocorticoids. BioFactors 3, 97-102 (1991).

[3] H. Bisgaard, J. K. Kristensen and J. Sondergaard, A new 
technique for ranking vascular corticosteroid effects in humans using laser-Doppler velocimetry. J. Invest. Dermatol. 86, 275-278 (1986).

[4] R. Marks and M. Sawyer, Glucocorticoid-induced vasoconstriction in human skin. An inhibitory role on phospholipase A2 activity. Arch. Dermatol. 122, 881-883 (1986).
[5] M. W. Radomski, R. M. Palmer and S. Moncada, Glucocorticoids inhibit the expression of an inducible, but not the constitutive, nitric oxide synthase in vascular endothelial cells. Proc. Natl. Sci. USA, 87, 10043-10047 (1990). 